\title{
Design of unknown input fuzzy observer for vehicle dynamics estimation with road geometry consideration
}

\author{
H. Dahmani M. Chadli A. Rabhi A. El Hajjaji \\ "Laboratoire Modélisation, Information et Systèmes", UPJV-MIS (EA4290), \\ 7 Rue Moulin Neuf, 80000 Amiens, France
}

\begin{abstract}
A methodology for estimating vehicle dynamics whith road geometry consideration is presented in this paper. Vehicle sideslip and roll parameters are estimated in the presence of the road bank angle and the road curvature as unknown inputs. The unknown inputs are then estimated using the observer results. This information about the road geometry and vehicle parameters is needed in the driver assistance systems to be able to calculate the risk index of a rollover or a lane departure crashes. Taking into account the unmeasured variables, an unknown inputs (TS) observer is then designed on the basis of the measure of the roll rate, the steering angle and the lateral offset given by the distance between the road centerline and the vehicle axe at a look-ahead distance. Synthesis conditions of the proposed fuzzy observer are formulated in terms of Linear Matrix Inequalities (LMI) using Lyapunov method. Simulation results show good efficiency of the proposed method to estimate both vehicle dynamics and road geometry.
\end{abstract}

Keywords: Unknown input fuzzy observer, vehicle dynamics, road geometry estimation, LMI.

\section{Introduction}

The possibility to accurately measure or estimate vehicle dynamics and road geometry is a critical determinant in the performance of many vehicle control and driver assistance systems such as braking control, lane departure avoidance, rollover detection and lateral control systems [1][2]. In emergency situations, sideslip is necessary to detect a sliding vehicle, the road curvature is necessary to detect a lane departure, vehicle roll and road bank angle are very useful to detect a rollover. Vehicle parameters and road geometry are necessary in determining the vehicle's behavior and the crash risk in such situations. However, all these values are not directly measured on production cars because the adequate sensors and measurement systems still very expensive. Therefore they must be estimated using available measurements [3].

Over the last few years, several methods were proposed to estimate the vehicle dynamics, some methods propose to integrate inertial sensors directly, others use a physical vehicle model to design a model based observer [4][5]. A combination or switch between these two methods was also used in order to estimate vehicle parameters [1][3]. Direct integration methods can accumulate sensor errors and unwanted measurements from road grade and superelevation. Methods based on a physical vehicle model can be sensitive to changes in the vehicle parameters but this effect can be reduced using uncertain and robust observers.

The problem of observer design with unknown inputs has received considerable attention during the two last decades [6][7]. In [8] a Proportional- Integral (PI) observer is proposed to estimate the state and the fault simultaneously. The presence of the integral of the estimation error makes it effective in estimating the system perturbations and input disturbances. However, the performance of these kinds of observers are generally deteriorated in the presence of measurement noise [9]. In [10] the authors propose a descriptor system based approach for a PI observer design in order to estimate the lateral vehicle dynamics in combination with the estimation of possible yaw moment disturbance input, wind gust input or road bank input. A paper given in [11] presents a method for identifying road bank and vehicle roll separately using a disturbance observer and a vehicle dynamic model. But these methods are only reliable in the linear region because they consider a linear approximation of the cornering forces. In this work a model based unknown input observer is designed in order to estimate both vehicle dynamics and road geometry (road curvature and road bank angle), a vision system (camera) is used to measure the lateral displacement at a lookahead distance of the vehicle. A representation of the nonlinear model of vehicle lateral and roll dynamics by a Takagi-Sugeno (TS) fuzzy model [12], has been considered. This representation has been largely used and studied over the last few years (see for example [13]).

This paper is organized as follows: section II introduces the used vehicle dynamics model and its representation by a TS fuzzy model. Section III focuses on the TS observer designed by considering the case where the activation functions depend on 


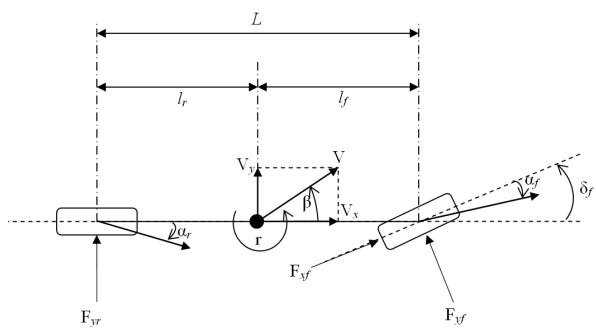

Figure 1: Vehicle lateral parameters

unmeasured variables. The estimation of the road curvature and the road bank angle is also presented in this section. Simulation results are presented in section IV. Finally, conclusions are given in section $\mathrm{V}$.

\section{VEHICLE MODEL DESCRIPTION}

The model used in this work describes vehicle lateral and roll dynamics, which is obtained by considering the well known single-track (bicycle) model with a roll degree of freedom (Fig. 1). The threedimensional model with road bank angle consideration and nonlinear tire characteristics of the four wheels vehicle behavior can be described by the following differential equations [11] [14]:

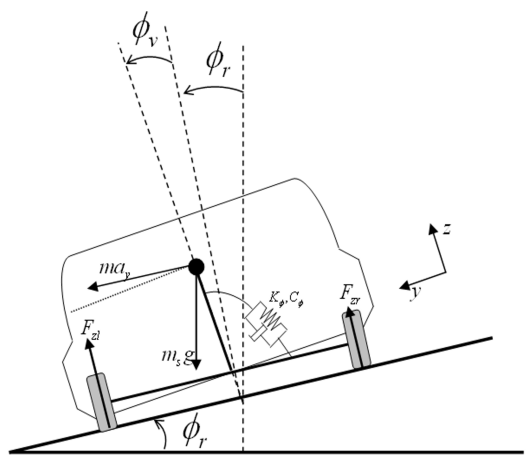

Figure 2: Vehicle roll parameters

$$
\left\{\begin{array}{l}
m\left(v \dot{\beta}+v \dot{\psi}+\ddot{\phi_{v}} h\right)=2 F_{y f}+2 F_{y r}-m_{s} g \phi_{r} \\
I_{z} \ddot{\psi}=2 F_{y f} l_{f}-2 F_{y r} l_{r} \\
I_{x} \ddot{\phi_{v}}=m_{s} g h\left(\phi_{v}+\phi_{r}\right)+m_{s} a_{y} h-K_{\phi} \phi_{v}-C_{\phi} \dot{\phi_{v}}
\end{array}\right.
$$

Where $\beta$ denotes the side slip angle, whereas $\psi$, $\phi_{v}$ and $\phi_{r}$ are respectively the vehicle yaw, the roll angle and the road bank angle, $F_{f}$ is the cornering force of the two front tires, $F_{r}$ is the cornering force of the two rear tires. For further description of the parameters appearing in the vehicle dynamics model refer to table 1 .

\subsection{TS representation of the the cornering forces}

In this work, we take into account the nonlinear forces by considering a TS representation of the tire model using the following rules:

If $\left|\alpha_{f}\right|$ is $M_{1}$ then $\left\{\begin{array}{l}F_{y f}=C_{f 1} \alpha_{f} \\ F_{y r}=C_{r 1} \alpha_{r}\end{array}\right.$

If $\left|\alpha_{f}\right|$ is $M_{2}$ then $\left\{\begin{array}{l}F_{y f}=C_{f 2} \alpha_{f} \\ F_{y r}=C_{r 2} \alpha_{r}\end{array}\right.$

$C_{f i}, C_{r i}$ are the front and rear tire cornering stiffness which depend of the road adhesion and the mass of vehicle.

The slip angle of the front tires $\alpha_{f}$ and the slip angle of the rear tires $\alpha_{r}$ are given as follow:

$$
\begin{aligned}
& \alpha_{f}=\delta_{f}-\frac{l_{f} \dot{\psi}}{v}-\beta \\
& \alpha_{r}=\frac{l_{r} \dot{\psi}}{v}-\beta
\end{aligned}
$$

where $\delta_{f}$ is the front steer angle.

The overall forces are obtained by :

$$
\left\{\begin{array}{c}
F_{y f}=\mu_{1}\left(\left|\alpha_{f}\right|\right) C_{f 1} \alpha_{f}+\mu_{2}\left(\left|\alpha_{f}\right|\right) C_{f 2} \alpha_{f} \\
F_{y r}=\mu_{1}\left(\left|\alpha_{f}\right|\right) C_{r 1} \alpha_{r}+\mu_{2}\left(\left|\alpha_{f}\right|\right) C_{r 2} \alpha_{r}
\end{array}\right.
$$

Where $\mu_{j}(j=1,2)$ is the $j^{\text {th }}$ bell curve membership function of fuzzy set $M_{j}$. They satisfy the following properties:

$$
\left\{\begin{array}{l}
\sum_{i=1}^{2} \mu_{i}\left(\left|\alpha_{f}\right|\right)=1 \\
0 \leq \mu_{i}\left(\left|\alpha_{f}\right|\right) \leq 1 \forall i=1,2
\end{array}\right.
$$

The expressions of the membership functions used are:

$$
\begin{gathered}
\mu_{i}\left(\left|\alpha_{f}\right|\right)=\frac{\omega_{i}\left(\left|\alpha_{f}\right|\right)}{\sum_{i=1}^{2} \omega_{i}\left(\left|\alpha_{f}\right|\right)}, \quad i=1,2 \\
\text { where } \omega_{i}\left(\left|\alpha_{f}\right|\right)=\frac{1}{\left(1+\left|\left(\frac{\left|\alpha_{f}\right|-c_{i}}{a_{i}}\right)\right|\right)^{2 b_{i}}}
\end{gathered}
$$

Table 1: Vehicle model parameter definition

\begin{tabular}{cll}
\hline Parameter & Description & Unit \\
\hline$\beta$ & Sideslip angle at CG & {$[\mathrm{rad}]$} \\
$\dot{\psi}$ & Yaw rate & {$[\mathrm{rad} / \mathrm{s}]$} \\
$\phi_{v}$ & Roll angle & {$[\mathrm{rad}]$} \\
$\dot{\phi_{v}}$ & Roll rate & {$[\mathrm{rad} / \mathrm{s}]$} \\
$\delta_{f}$ & Front ster angle & {$[\mathrm{rad}]$} \\
$m_{s}$ & Sprung vehicle mass & {$[\mathrm{kg}]$} \\
$m$ & Vehicle mass & {$[\mathrm{kg}]$} \\
$v$ & Vehicle speed & {$[\mathrm{m} / \mathrm{s}]$} \\
$I_{x}$ & Roll moment of inertia at CG & {$\left[\mathrm{kgm}{ }^{2}\right]$} \\
$I_{z}$ & Yaw moment of inertia at CG & {$\left[\mathrm{kgm}{ }^{2}\right]$} \\
$l_{r}$ & Distance from CG to rear axle & {$[\mathrm{m}]$} \\
$l_{f}$ & Distance from CG to front axle & {$[\mathrm{m}]$} \\
$T$ & Vehicle track width & {$[\mathrm{m}]$} \\
$h$ & CG height from roll axis & {$[\mathrm{m}]$} \\
$C_{\phi}$ & Combined roll damping coefficient & {$[\mathrm{Nms} / \mathrm{rad}]$} \\
$k_{\phi}$ & Combined roll stifness coefficient & {$[\mathrm{Nm} / \mathrm{rad}]$} \\
\hline
\end{tabular}

\subsection{TS model with vision system measurement}

Using a vision system measuring the lateral displacement of the vehicle at a look-ahead distance 
(Fig. 3), the equations describing the evolution of the measurement extracted from image, caused by the motion of the car and changes in the road geometry can be written as follows [15]:

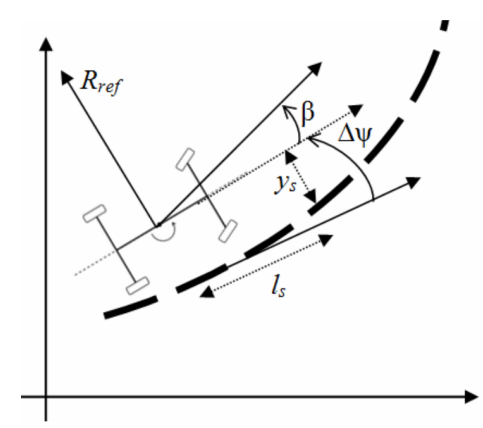

Figure 3: Vision System measurement

$$
\dot{y}_{s}=v(\beta+\Delta \psi)+l_{s} \Delta \dot{\psi}
$$

The angular displacement $\Delta \psi$ is obtained as follows:

$$
\Delta \dot{\psi}=\dot{\psi}-\frac{v}{R_{c}}=\dot{\psi}-v w
$$

$y_{s}$ is the offset from the centerline at the lookahead distance, $\Delta \psi$ the angle between the tangent to the road and the orientation of the vehicle with respect to the road, $l_{s}$ the look-ahead distance at which the measurement is taken and $w$ the road curvature.

Using the above approximation idea of nonlinear lateral forces by TS rules the vehicle dynamics model (1) and the vision dynamics (6) and (7) leads to a single dynamical system with the following form:

$$
\begin{aligned}
\dot{x}(t) & =\sum_{i=1}^{2} \mu_{i}\left(\left|\alpha_{f}\right|\right)\left(A_{i} x(t)+B_{i} \delta_{f}(t)\right)+B_{f} f(t) \\
y(t) & =C x(t)
\end{aligned}
$$

with

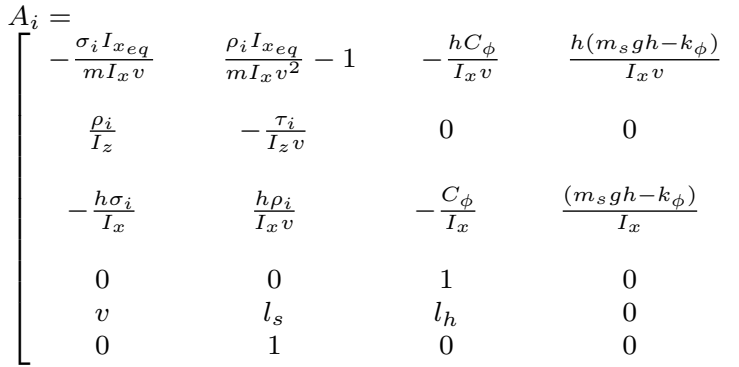$$
B_{i}=\left[\begin{array}{llllll}
2 \frac{C_{f i} I_{x_{e q}}}{m I_{x} v} & 2 \frac{C_{f i} l_{f}}{I_{z}} & 2 \frac{m_{s} h C_{f i}}{m I_{x}} & 0 & 0 & 0
\end{array}\right]^{T}
$$$$
B_{f}=\left[\begin{array}{cccccc}
-\frac{m_{s} g}{m v} & 0 & 0 & 0 & 0 & 0 \\
0 & 0 & 0 & 0 & -l_{s} v & -v
\end{array}\right]^{T} \text {, }
$$$$
C=\left[\begin{array}{llllll}
0 & 1 & 0 & 0 & 0 & 0 \\
0 & 0 & 0 & 0 & 1 & 0
\end{array}\right], f(t)=\left[\begin{array}{ll}
\phi_{r}(t) & w(t)
\end{array}\right]^{T}
$$$$
\text { and } x(t)=\left[\begin{array}{llllll}
\beta(t) & \dot{\psi}(t) & \dot{\phi}(t) & \phi(t) & y_{s}(t) & \Delta \psi(t)
\end{array}\right]^{T}
$$
is the state vector of the model.

Where $I_{x_{e q}}$ denotes the equivalent roll moment of inertia of the vehicle about the roll axis, which is given by

$$
I_{x_{e q}}=I_{x}+m_{s} h^{2}
$$

and $\sigma_{i}, \rho_{i}$ and $\tau_{i}$ are auxiliary variables introduced in order to simplify the model description, they are defined as follows:

$\sigma_{i}=2\left(C_{r i}+C_{f i}\right), \quad \rho_{i}=2\left(l_{r} C_{r i}-l_{f} C_{f i}\right), \quad \tau_{i}=2\left(l_{f}^{2} C_{f i}+l_{r}^{2} C_{r i}\right)$

\section{Vehicle dynamics and road geometry estimation}

To estimate the road curvature, it is necessary to know some of the model states which are difficult or even very expensive to measure directly. These latter can be estimated using measurable signals such as the lateral displacement and the steering angles. In this study, we design a TS model based observer which estimates the sideslip angle, the yaw rate, the relative yaw rate and the vehicle roll angle by considering the road curvature $w(t)$ and the road bank angle as unknown inputs (Fig. 4). We measure steering angle, vehicle velocity, the roll rate and the lateral displacement at a lookahead distance provided by a camera.

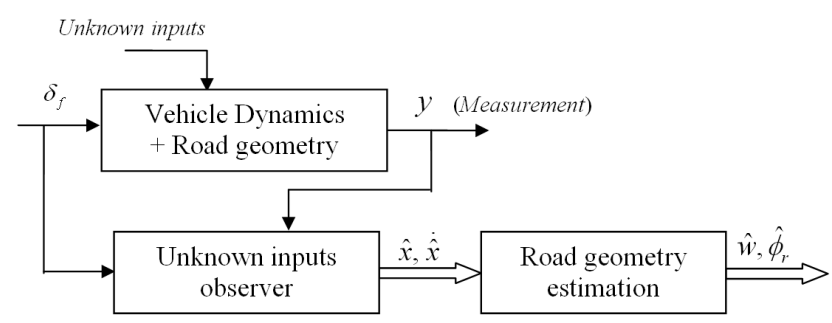

Figure 4: Vehicle dynamics and road geometry estimation

\subsection{TS Fuzzy observer design conditions}

The TS model based estimator in presence of the road curvature as an unknown input is represented as follows:

$$
\begin{aligned}
& \dot{\hat{x}}(t)=\sum_{i=1}^{2} \mu_{i}\left(\left|\hat{\alpha_{f}}\right|\right)\left(A_{i} \hat{x}(t)+B_{i} \delta_{f}(t)+L_{i}(y(t)-\hat{y}(t))\right)+\eta(t) \\
& \hat{y}(t)=C \hat{x}(t)
\end{aligned}
$$

The aim of the design is to determine gain matrices $L_{i}$, and variable $\eta(t) \in \Re^{n}$, that guarantee the asymptotic convergence of $\hat{x}(t)$ towards $x(t)$.

Notice that the variable $\eta(t)$ is introduced to compensate the errors due to the used unmeasurable premise variables in the dynamic of the state estimation error. Let us define the state estimation error:

$$
e(t)=x(t)-\widehat{x}(t)
$$


The output estimation error is defined as follows:

$$
e_{y}(t)=y(t)-\hat{y}(t)=C(x(t)-\hat{x}(t))=C e(t)
$$

In the following sections, to simplify the expression of equations, time variable $(t)$ will be omitted.

The dynamic of the state estimation error is governed by:

$\dot{e}=\sum_{i=1}^{2} \mu_{i}\left(\left|\hat{\alpha_{f}}\right|\right)\left(A_{i}-L_{i} C\right) e+\Delta A x+\Delta B \delta_{f}+B_{f} f-\eta$

where

$\Delta A=\sum_{i=1}^{2} \bar{\mu}_{i} A_{i}, \quad \Delta B=\sum_{i=1}^{2} \bar{\mu}_{i} B_{i}, \quad \bar{\mu}_{i}=\mu_{i}\left(\left|\alpha_{f}\right|\right)-\mu_{i}\left(\left|\hat{\alpha_{f}}\right|\right)$

which can be rewritten as follows:

$$
\dot{e}=\sum_{i=1}^{2} \mu_{i}\left(\left|\hat{\alpha_{f}}\right|\right)\left(A_{i}-L_{i} C\right) e+\Delta A x+\bar{B}_{f} \bar{f}-\eta
$$

where $\bar{B}_{f}=\left[\begin{array}{ll}B_{f} & I\end{array}\right] \quad \bar{f}=\left[\begin{array}{ll}f & b\end{array}\right]^{T}$ and $b=$ $\Delta B^{T} \delta_{f}$

Remark 1: The convex sum property of the membership functions allows to write

$$
-1 \leq \bar{\mu}_{i} \leq 1
$$

then the variable matrices $\Delta A$ and $\Delta B$ are bounded and the following property is verified

$$
\|\Delta A\| \leq \rho \quad, \quad \rho=\sum_{i=1}^{2} \rho_{i}
$$

where $\rho_{i}>0$ is the matrix norm of $A_{i}$. The TS estimator gains have been computed by considering the effect of the unknown input vector $\bar{f}$ to the state estimation errors. One possible method is to minimize the $\mathcal{L}_{2}$ gain (H-infinity norm) from $\bar{f}$ to estimation errors.

The $\mathcal{L}_{2}$ gain between the unknown input $\bar{f}$ and the estimation error $e$ is defined by the following quantity:

$$
\gamma=\sup \frac{\|e\|}{\|\bar{f}\|}
$$

By the definition of the supremum and the $\mathcal{L}_{2}$ gain, (19) can be expressed as

$$
\int_{0}^{\infty} e^{T}(t) e(t) d t \leq \gamma^{2} \int_{0}^{\infty} \bar{f}^{T}(t) \bar{f}(t) d t
$$

The demonstration of the asymptotic convergence of the robust observer uses the following lemma:

Lemma 1: For any matrices $X$ and $Y$ with appropriate dimensions, the following property holds for any positive scalar $\beta$ :

$$
X^{T} Y+Y^{T} X \leq \beta X^{T} X+\beta^{-1} Y^{T} Y
$$

Theorem 1: The system (14) is stable and the $\mathcal{L}_{2}$ gain of the transfer from the unknown input vector $\bar{f}$ to the state estimation errors is bounded by $\gamma$ if there exist a positive and symmetric matrix $X$, matrices $N_{i}$, and positive scalars $\beta_{0}$ and $\beta_{1}$ such that the following LMIs hold for $i=1,2$ :

$$
\left[\begin{array}{ccc}
\Omega_{i} & X \bar{B}_{f} & X \\
\bar{B}_{f}^{T} X & -\gamma^{2} I & 0 \\
X & 0 & -\beta_{1} I
\end{array}\right]<0
$$

with $\Omega_{i}=A_{i}^{T} X+X A_{i}+N_{i} C+C^{T} N_{i}^{T}+\beta_{0} \rho^{2} I$ and $\eta$ is given by the following equations

$\left\{\begin{array}{l}\eta=0 \quad \text { if } \quad e_{y}=0 \\ \eta=\left(\frac{\beta_{1} \beta_{0}}{\beta_{1}-\beta_{0}}\right) \rho^{2} \frac{\widehat{x}(t)^{T} \widehat{x}(t)}{2 e_{y}(t)^{T} e_{y}(t)} X^{-1} C^{T} e_{y}(t) \quad \text { otherwise }\end{array}\right.$

The TS estimator gains are then computed by

$$
L_{i}=X^{-1} N_{i}
$$

Proof: Consider the following Lyapunov function candidate:

$$
V(t)=e^{T}(t) X e(t)
$$

where $X=X^{T}>0$. Substituting (14) into (25) and taking the time derivative gives

$$
\begin{gathered}
\dot{V}=\sum_{i=1}^{2} \mu_{i}\left(\left|\hat{\alpha_{f}}\right|\right) e^{T}\left(\bar{A}_{i}^{T} X+X \bar{A}_{i}\right) e+x^{T} \Delta A^{T} X e+e^{T} X \Delta A x- \\
2 \eta^{T} X e+2 e^{T} \bar{B}_{f} \bar{f}
\end{gathered}
$$

where $\bar{A}_{i}=A_{i}-L_{i} C$. The lemma (1) property allows to write:

$$
\begin{gathered}
\dot{V} \leq \sum_{i=1}^{2} \mu_{i}\left(\left|\hat{\alpha_{f}}\right|\right) e^{T}\left(\bar{A}_{i}^{T} X+X \bar{A}_{i}+\beta_{1}^{-1} X^{2}\right) e-2 \eta^{T} X e+ \\
\beta_{1} \rho^{2}\left(\hat{x}^{T} \hat{x}+e^{T} e\right)+\beta_{1} \rho^{2}\left(\hat{x}^{T} e+e^{T} \hat{x}\right)+2 e^{T} \bar{B}_{f} \bar{f}
\end{gathered}
$$

Using again the property of the lemma (1), the expression (27)

$$
\begin{gathered}
\dot{V} \leq \sum_{i=1}^{2} \mu_{i}\left(\left|\hat{\alpha_{f}}\right|\right) e^{T}\left(\bar{A}_{i}^{T} X+X \bar{A}_{i}+\beta_{1}^{-1} X^{2}+\beta_{0} \rho^{2} I\right) e- \\
2 \eta^{T} X e+\beta_{1}\left(1+\beta_{2}\right) \rho^{2} \hat{x}^{T} \hat{x}+2 e^{T} \bar{B}_{f} \bar{f}
\end{gathered}
$$

with $\beta_{0}=\beta_{1}\left(1+\beta_{2}^{-1}\right)$. Substituting variable $\eta$ by the expression given by (23), allows to write :

$-2 \eta^{T} X e=\left(\frac{\beta_{1} \beta_{0}}{\beta_{1}-\beta_{0}}\right) \rho^{2} \frac{\hat{x}^{T} \hat{x}}{e_{y}^{T} e_{y}} e_{y}^{T} C X^{-1} X e=\beta_{1}\left(1+\beta_{2}\right) \rho^{2} \hat{x}^{T} \hat{x}$

Therefore, after simplification, we obtain:

$\dot{V} \leq \sum_{i=1}^{2} \mu_{i}\left(\left|\hat{\alpha_{f}}\right|\right) e^{T}\left(\bar{A}_{i}^{T} X+X \bar{A}_{i}+\beta_{1}^{-1} X^{2}+\beta_{0} \rho^{2} I\right) e+2 e^{T} \bar{B}_{f} \bar{f}$

The system (14) is stable and the $\mathcal{L}_{2}$ gain of the transfer from the unknown input vector $\bar{f}$ to the state estimation errors is bounded by $\gamma$ if the following condition holds

$$
J_{\infty}=\dot{V}+e^{T} e-\gamma^{2} \bar{f}^{T} \bar{f}<0
$$


substituting (30) in (31), the following inequality is obtained

$$
\begin{gathered}
\sum_{i=1}^{2} \mu_{i}\left(\left|\hat{\alpha_{f}}\right|\right) e^{T}\left(\bar{A}_{i}^{T} X+X \bar{A}_{i}+\beta_{1}^{-1} X^{2}+\beta_{0} \rho^{2} I\right) e+2 e^{T} \bar{B}_{f} \bar{f}+ \\
e^{T} e-\gamma^{2} \bar{f}^{T} \bar{f}<0
\end{gathered}
$$

Inequality (32) can be rearranged as follows:

$$
\sum_{i=1}^{2} \mu_{i}\left(\left|\hat{\alpha_{f}}\right|\right)\left(\left[\begin{array}{c}
e^{T} \\
\bar{f}^{T}
\end{array}\right]^{T}\left[\begin{array}{cc}
\Gamma_{i} & X \bar{B}_{f} \\
\bar{B}_{f}^{T} X & -\gamma^{2} I
\end{array}\right]\left[\begin{array}{l}
e \\
\bar{f}
\end{array}\right]\right)<0
$$

where $\Gamma_{i}=A_{i}^{T} X+X A_{i}-C^{T} L_{i}^{T} X-X L_{i} C+$ $\beta_{1}^{-1} X^{2}+\beta_{0} \rho_{i}^{2} I+I$

Since the conditions in (33) are not convex in variables $X$ and $L_{i}$, by introducing a new variable, $N_{i}=X L_{i}$ and applying the Schur complement, (33) can be rewritten as the equivalent LMI condition (22).

The solution of this LMI in $X$ and $N_{i}$ allows to compute the observer gains $L_{i}=X^{-1} N_{i}, \quad \beta_{1}, \quad \beta_{0}$ and then $\eta$ which define completely the observer (11).

\subsection{Road curvature and road bank angle estimation}

Once the states of the system rebuilt, they will be used to estimate the road curvature. From equation (6), the road curvature $w$ can be computed as follows:

$$
\tilde{w}=\frac{1}{l_{s}} \hat{\beta}+\frac{1}{l_{s}} \Delta \hat{\psi}+\frac{1}{v} \dot{\hat{\psi}}-\frac{1}{l_{s} v} \dot{y}_{s}
$$

where $v$ is the vehicle velocity, $\dot{\hat{\psi}}$ and $\Delta \dot{\hat{\psi}}$ are the estimate results of the observer.

From equation (8), the road bank angle is estimated as follow:

$$
\tilde{\phi}_{r}(t)=\left(-\frac{m v}{m_{s} g}\right)(\dot{\hat{\beta}}(t)-s(t))
$$

with

$$
\begin{gathered}
s(t)=\sum_{i=1}^{2} \mu_{i}\left(\left|\hat{\alpha_{f}}\right|\right)\left(A_{11 i} \hat{\beta}(t)+A_{12 i} \dot{\hat{\psi}}(t)+A_{13 i} \dot{\hat{\phi}}_{v}(t)+\right. \\
\left.A_{14 i} \hat{\phi}_{v}(t)+B_{1 i} \delta_{f}(t)\right)
\end{gathered}
$$

\section{Simulation results}

In this study, simulations are conducted to illustrate the efficiency of the above presented method. The vehicle model and the designed observer are implemented in MATLAB software. The simulated vehicle test is in two successive turns, the vehicle path for this scenario is shown in Fig. 5. The road curvature and the road bank angle are illustrated in Fig. 7. Table 2 gives the vehicle parameter values.

The estimated vehicle states using the designed observer are shown in Fig. 6. It is clearly shown that the observer converge fatly to the actual states dispite the initial conditions of the model.

Figure 7 shows the road curvature and the road bank angle estimation. This results show the performance of the method to estimate simultaneously
Table 2: Simulation vehicle parameters

\begin{tabular}{cll}
\hline Parameter & value & Unit \\
\hline$m_{s}$ & 1592 & {$[\mathrm{~kg}]$} \\
$m$ & 1832 & {$[\mathrm{~kg}]$} \\
$v$ & 20 & {$[\mathrm{~m} / \mathrm{s}]$} \\
$I_{x}$ & 614 & {$\left[\mathrm{kgm}^{2}\right]$} \\
$I_{z}$ & 2488 & {$\left[\mathrm{kgm}^{2}\right]$} \\
$l_{r}$ & 1.77 & {$[\mathrm{~m}]$} \\
$l_{f}$ & 1.18 & {$[\mathrm{~m}]$} \\
$T$ & 1.5 & {$[\mathrm{~m}]$} \\
$h$ & 0.559 & {$[\mathrm{~m}]$} \\
$C_{\phi}$ & 6000 & {$[\mathrm{Nms} / \mathrm{rad}]$} \\
$k_{\phi}$ & 48000 & {$[\mathrm{Nm} / \mathrm{rad}]$} \\
\hline
\end{tabular}

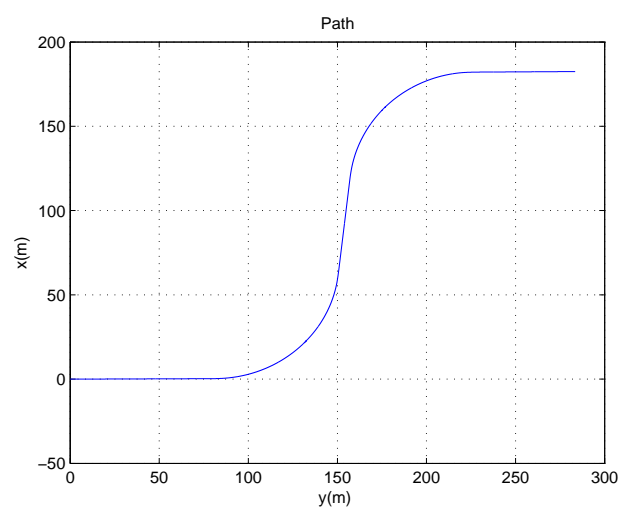

Figure 5: The vehicle path

the vehicle dynamics and the road geometry. But it still not completely ready to be tested in experimental studies since the vehicle speed, parameters variations and sensor noise are not considered. This issue will be considered in the future works before performing real world

\section{CONCLUSIONS AND FUTURE WORKS}

Using $H_{\infty}$ approach, a Takagi-Sugeno fuzzy observer with unknown inputs is designed in presence of the road geometry. The nonlinear model given by vehicle lateral and roll dynamics is represented by a TS fuzzy model. This representation take into account the non-linearities introduced by the cornering forces. The vehicle dynamics and the road geometry are estimated simultaneously using available measurement like the offset displacement at a lookahead distance of the vehicle given by a camera. The designed TS observer use unmeasurable premise variables. Design conditions are formulated in LMI terms easy to solve using numerical tools. The conducted simulation show good performance of the designed observer and the used method to estimate vehicle dynamics and the road attributes. The advantage of this approach is in the used model which can be very useful in the lane departure de- 

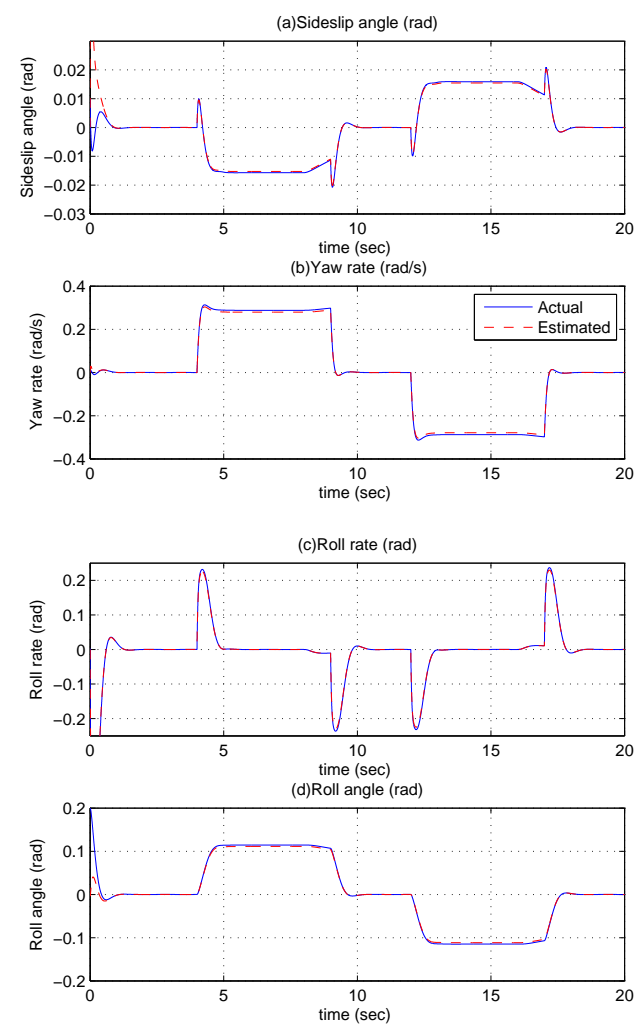

Figure 6: Simulation results of the vehicle state estimator

tection and rollover detection simultaneously. In further works we will extend the results by considering more complex vehicle model in order to increase the observer robustness. We also expect to validate our approach in experimental studies using software simulator and experimental vehicle.

\section{References}

[1] Bevly, D.M. Ryu, J. Gerdes, J.C. "Integrating INS Sensors With GPS Measurements for Continuous Estimation of Vehicle Sideslip, Roll, and Tire Cornering Stiffness". ITS, IEEE Transactions on vol.7, pp. 483-493, 2006.

[2] H. Dahmani, M. Chadli, A. Rabhi and A.El Hajjaji Road angle considerations for detection of impending vehicle rollover, IFAC AAC 2010, 12-14 july 2010, Munich, Germany.

[3] A. Nishio, et. al. "Development of Vehicle Stability Control System Based on Vehicle Sideslip Angle Estimation". SAE Paper No. 2001-010137.

[4] J. Farrelly and P. Wellstead "Estimation of Vehicle Lateral Velocity". IEEE Conference on Control Applications 1996, p.552-557.

[5] J. Farrelly and P. Wellstead "Slip-Angle Estimation for Vehicle Stability Control". Veh. Sys. Dyn. 1999, v.32, no.4, p.375-388.

[6] M. Hou and P.C. Muller. "Observer design for descriptor systems". IEEE Transaction in Au-
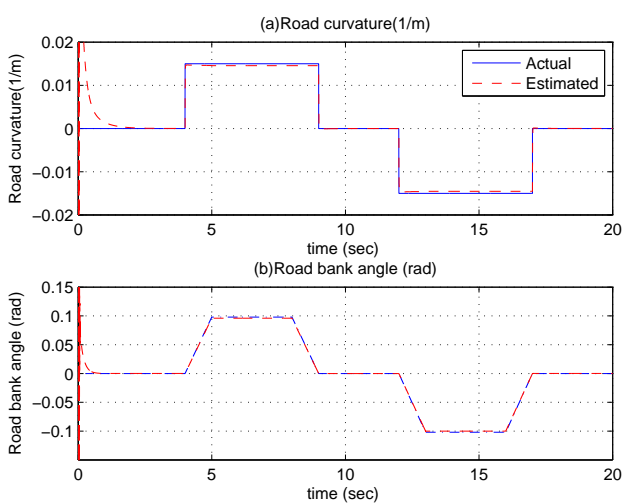

Figure 7: The road geometry estimation

tomatic Control vol. 44, pp. 164-169, 1999.

[7] M. Chadli, A. Akhenak, J. Ragot, and D. Maquin. "State and Unknown Input Estimation for Discrete Time Multiple Model". Journal of the Franklin Institute, Vol. 346, No. 6, pp. 593610, 2009.

[8] D. Koenig. "Unknown input proportional multiple-integral observer design for linear descriptor systems: Application for state and fault estimation". IEEE Trans. Automat. Contr. vol 50, pp. 212-217, 2005.

[9] Z. Gao, D. W. C. Ho. "Proportional multipleintegral observer design for descriptor systems with measurement output disturbances". IEE Proc.-Control Theory Appl. vol 151, 3, 2004.

[10] S. Mammar, S. Glaser, M. Netto "Vehicle Lateral Dynamics Estimation using Unknown Input Proportional-Integral Observers". Proceedings of the 2006 American Control Conference Minneapolis, Minnesota, USA, June 14-16, 2006.

[11] J. Ryu and J. Christian Gerdes. "Estimation of Vehicle Roll and Road Bank Angle". In Proc. of the 2004 American Control Conference.,Boston, Massachusetts June 30. July 2.2004.

[12] T.Takagi and M. Sugeno. "Fuzzy identification of systems and its applications to modeling and control". IEEE Transactions on Systems, Man, and Cybernetics vol.15, pp. 116-132, 1985.

[13] K. Tanaka, T. Ikeda, and H. Wang, Fuzzy regulators and fuzzy observers, Relaxed stability conditions and lmi-based designs, IEEE TFS, vol. 6 , no. 2, pp. 250-265, 1998.

[14] S. Kidane, L. Alexander, R. Rajamani, P. Starr and M. Donath. "Road Bank Angle Considerations in Modeling and Tilt Stability Controller Design for Narrow Commuter Vehicles". In Proc. of the 2006 ACC., Minneapolis, Minnesota, USA, June 14-16, 2006

[15] H. Dahmani, M. Chadli, A. Rabhi and A.El Hajjaji Fuzzy uncertain observer with unknown inputs for Lane departure detection, American Control Conference ACC 2010 June 30 - July 2, 2010 Maryland, USA. 